\title{
Neuroprotectin D1 attenuates brain damage induced by transient middle cerebral artery occlusion in rats through TRPC6/CREB pathways
}

\author{
CHENGYE YAO*, JIANCHENG ZHANG ${ }^{*}$, FANG CHEN and YUN LIN \\ Department of Anesthesiology, Union Hospital, Tongji Medical College, \\ Huazhong University of Science and Technology, Wuhan, Hubei 430022, P.R. China
}

Received January 3, 2013; Accepted May 31, 2013

DOI: $10.3892 / \mathrm{mmr} .2013 .1543$

\begin{abstract}
Neuroprotectin D1 (NPD1) may serve an endogenous neuroprotective role in brain ischemic injury, yet the underlying mechanism involved is poorly understood. In the present study, we aimed to investigate whether intracerebroventricular (ICV) injection of NPD1 is neuroprotective against transient focal cerebral ischemia. We also sought to verify the neuroprotective mechanisms of NPD1. Rats subjected to $2 \mathrm{~h}$ ischemia followed by reperfusion were treated with NPD1 at $2 \mathrm{~h}$ after reperfusion. PD98059 was administered $20 \mathrm{~min}$ prior to surgery. Western blot analysis was performed to detect the protein levels of calpain-specific aII-spectrin breakdown products of $145 \mathrm{kDa}$ (SBDP145), transient receptor potential canonical (subtype) 6 (TRPC6) and phosphorylation of $\mathrm{cAMP} / \mathrm{Ca}^{2+}$-response element binding protein (p-CREB) at 12, 24 and $48 \mathrm{~h}$ after reperfusion. The immunoreactivity of p-CREB and TRPC6 was measured by quantum dot-based immunofluorescence analysis. Infarct volume and neurological scoring were evaluated at $48 \mathrm{~h}$ after reperfusion. NPD1, when applied at $2 \mathrm{~h}$ after reperfusion, significantly reduced infarct volumes and increased neurological scores at $48 \mathrm{~h}$ after reperfusion, accompanied by elevated TRPC6 and p-CREB activity, and decreased SBDP145 activity. When mitogen-activated protein kinase kinase (MEK) activity was specifically inhibited, the neuroprotective effect of NPD1 was attenuated and correlated with decreased CREB activity. Our results clearly showed that ICV injection of NPD1 at $2 \mathrm{~h}$ after reperfusion improves the neurological status of middle
\end{abstract}

Correspondence to: Dr Yun Lin, Department of Anesthesiology, Union Hospital, Tongji Medical College, Huazhong University of Science and Technology, 1277 Jiefang Avenue, Wuhan, Hubei 430022, P.R. China

E-mail: linyun7801@yahoo.com.cn

${ }^{*}$ Contributed equally

Key words: neuroprotectin D1, neuroprotection, middle cerebral artery occlusion, TrpC, CREB, rat cerebral artery occlusion (MCAO) rats through the inhibition of calpain-mediated TRPC6 proteolysis and the subsequent activation of CREB via the Ras/MEK/ERK pathway.

\section{Introduction}

Acute ischemic stroke, the most common form of stroke, can produce an irreversibly injured core, in which cell death is rapid and not salvageable, and an ischemic penumbra, where tissue is damaged but potentially salvageable $(1,2)$. The penumbra has a limited life span and appears to undergo irreversible damage within a few hours unless reperfusion is initiated and/or neuroprotective therapy is administered (3). Therefore, the early rapid recovery of cerebral blood flow and effective neuroprotective treatment in cerebral ischemia remains vital. However, to date, there remains no established treatment for lessening ischemic brain injury.

Brain injury after focal cerebral ischemia develops from a series of pathological processes, including excitotoxicity, peri-infarct depolarizations, ionic imbalance, oxidative stresses and apoptosis $(1,2,4,5)$. Although these mechanisms have been implicated in ischemic neuronal death, $\mathrm{Ca}^{2+}$ overload remains the central focus. Cerebral ischemia causes significant glutamate release and exposure to high levels of glutamate leads to the overactivation of N-methyl-D-aspartate receptors (NMDARs), causing $\mathrm{Ca}^{2+}$ overload, which leads to calpain activation (5-7). The activation of calpain leads to proteolysis of transient receptor potential canonical (subtype) 6 (TRPC6) channels. TRPC6 channels play a critical role in promoting neuronal survival against focal cerebral ischemia (8). TRPC6 activates cAMP-response element binding protein (CREB) through the Ras/MEK/ERK pathway, and contributes to TRPC6-mediated CREB activation, resulting in neuronal survival (9). Activation of calpain leads to TRPC6 degradation and contributes to neuronal damage in ischemia (8). Therefore, inhibition of TRPC6 degradation to preserve neuronal survival may be a new therapeutic strategy against ischemic brain damage.

NPD1, a stereospecific derivative of docosahexaenoic acid (DHA) formed through a lipoxygenase enzyme that acts on free DHA $(10,11)$, reduces infarct volume at $48 \mathrm{~h}$ after reperfusion (12). However, the precise mechanism responsible for the 
neuroprotective activity of NPD1 has yet to be fully elucidated. Potential mechanisms explaining how NPD1 may serve an endogenous neuroprotective role include reducing apoptosis, inhibiting leukocyte infiltration and pro-inflammatory gene expression, and binding toxic peroxides (12-16). However, previous studies have not unequivocally confirmed the effects of intracerebroventricular (ICV) injection of NPD1 on TRPC6/ CREB-mediated neuroprotection.

The present study was performed to investigate whether ICV injection of NPD1 at $2 \mathrm{~h}$ after reperfusion has a neuroprotective effect, and to verify whether NPD1 improves neurological status through inhibition of calpain proteolysis of TRPC6, subsequently inducing CREB activation via the Ras/ MEK/ERK pathway.

\section{Materials and methods}

Animals and surgical procedures. Male Sprague-Dawley rats, weighing 200-250 g, were purchased from Hunan weasleyg scene of experimental animals Co., Ltd. Experimental protocols were approved by the committee of experimental animals of Tongji Medical College and conformed to internationally accepted ethical standards (Guide for the care and use of laboratory animals; NIH Publication 80-23, revised 1978). The animals were anesthetized with $10 \%$ chloral hydrate (400 mg/kg, i.p.). Transient focal cerebral ischemia was produced by intraluminal occlusion of the right middle cerebral artery (MCA) for $2 \mathrm{~h}$. Briefly, the right carotid artery was exposed to separate the external carotid artery and the internal carotid artery. The external carotid artery was occluded at the level at which the MCA branches out and a 4-0 monofilament nylon suture (Beijing Sunbio Biotech Co. Ltd., Beijing, China) with a rounded tip was introduced through the internal carotid artery until mild resistance was felt. Two hours later, the filament was gently removed for the reperfusion (reperfusion confirmed by laser Doppler). Sham surgery rats were treated similarly, although the filament was not advanced to the origin of the MCA. The body temperature was maintained at $37.5 \pm 0.5^{\circ} \mathrm{C}$ with a temperature-controlled heating pad attached to a rectal probe during surgery. Continuous laser-Doppler flowmetry (Perimed PF5000, Stockholm, Sweden) was used to monitor regional cerebral blood flow (rCBF) in the cortex supplied by the MCA to ensure accurate occlusion and reperfusion. Animals that showed a CBF reduction $<70 \%$ were excluded from the experimental group, as well as animals that died after ischemia induction. In a separate experiment, physiological parameters (cranial temperature, arterial $\mathrm{pH}, \mathrm{PaCO}_{2}$ and $\mathrm{PaO}_{2}$ ) were monitored and analyzed ( $\left.\mathrm{n}=6\right)$. Arterial blood samples were obtained $5 \mathrm{~min}$ prior to ischemia (baseline), 60 min following ischemia, and 12, 24 and $48 \mathrm{~h}$ following reperfusion for blood gas analysis.

Animal groups and treatments. Rat ICV injection was performed under anesthesia with a stereotaxic instrument using a microsyringe pump. A scalp incision was made and a burr hole was opened in the right parietal skull, $1.8 \mathrm{~mm}$ lateral and $1.0 \mathrm{~mm}$ posterior to the bregma. A syringe was inserted into the brain to a depth of $4.2 \mathrm{~mm}$ below the cortical surface. Drugs or vehicle were injected slowly $(0.5 \mu \mathrm{l} / \mathrm{min})$ into the right ventricle.
All treatments were administered in a blinded manner. The rats were randomly divided into four groups, and each group was again divided into three subgroups ( $\mathrm{n}=12$ per subgroup) according to the time of reperfusion $(12,24$ and $48 \mathrm{~h}$ after reperfusion). The experimental groups and subgroups were as follows: i) Sham surgery (Group S; subgroup S12, S24 and S48); ii) middle cerebral artery occlusion (MCAO; Group I; subgroup I12, I24 and I48); iii) ischemia combined with NPD1 treatment (Group N; subgroup N12, N24 and N48) and iv) ischemia combined with NPD1 plus PD98059 (MEK inhibitor) treatment (Group P; subgroup P12, P24 and P48). Another 27 rats were randomly divided into three groups: i) Sham surgery (Group S); ii) MCAO (Group I); iii) ischemia combined with PD98059 treatment (Group M).

NPD1 (100 ng/ $/$ l; Cayman Chemical Company, Ann Arbor, MI, USA) was dissolved in ethanol. PD98059 $(1.5 \mathrm{mg} / \mathrm{ml}$; Sigma, St. Louis, MO, USA) was prepared in $1 \%$ DMSO (Sigma). NPD1 $(5 \mu \mathrm{l})$ or ethanol $(5 \mu \mathrm{l})$ was injected slowly $(0.5 \mu \mathrm{l} / \mathrm{min})$ into the right ventricle at $2 \mathrm{~h}$ after reperfusion. PD 98059 (0.5 ml, i.p.) or DMSO (0.5 ml, i.p.) was also administered to rats $20 \mathrm{~min}$ prior to the surgery.

Measurement of infarct volume. The extent of infarction was measured with 2,3,5-triphenyl-tetrazolium chloride (TTC). At $48 \mathrm{~h}$ after reperfusion, rats were deeply anesthetized with $10 \%$ chloral hydrate, and the brains were rapidly removed, washed in phosphate-buffered saline (PBS) at room temperature and frozen at $-20^{\circ} \mathrm{C}$ for $10 \mathrm{~min}$. Brain tissue from an area $4 \mathrm{~mm}$ anterior and $6 \mathrm{~mm}$ posterior to the bregma was cut into five serial $2 \mathrm{~mm}$ coronal sections. The sliced brain tissues were stained with 2\% TTC (Amresco, Solon, OH, USA) for $30 \mathrm{~min}$ at $37^{\circ} \mathrm{C}$ in the dark followed by overnight immersion in $4 \%$ paraformaldehyde in $0.1 \mathrm{M} \mathrm{PBS}, \mathrm{pH} 7.4$, at $4^{\circ} \mathrm{C}$. The infarcted tissue remained unstained (white), whereas normal tissue was stained red. The extent of ischemic infarction was traced and the integrated volume was calculated using Image $\mathbf{J}$ software (NIH Image). Infarct volume was calculated by adding the infarction areas of all sections and multiplying by slice thickness. To compensate for the effect of brain edema, the corrected infarct volume was calculated as follows: percentage of corrected infarct volume $=\{$ [total lesion volume - (ipsilateral hemisphere volume - contralateral hemisphere volume)]/ contralateral hemisphere volume\} x 100 .

Neurological test. Neurological evaluation of motor sensory functions was carried out at $48 \mathrm{~h}$ after reperfusion. An 18-point scale of neurologic deficit scores was used for evaluation of neurologic behavior (17). The scores were assessed in a blinded fashion. The scale was based on the following six tests: i) spontaneous activity; ii) symmetry in the movement of four limbs; iii) forepaw outstretching; iv) climbing; v) body proprioception; and vi) response to vibrissae touch. The score assigned to each rat at completion of the evaluation equaled the sum of all six test scores. The final minimum score was 3 and the maximum was 18 .

Lysis and protein content determination. All the rats were sacrificed by decapitation at 12,24 and $48 \mathrm{~h}$ after reperfusion. Slices containing maximal ischemic damage were selected (from an area between 3 and $6 \mathrm{~mm}$ posterior to the frontal 
Table I. Physiological parameters.

\begin{tabular}{lcccc}
\hline Time point & Temperature $\left({ }^{\circ} \mathrm{C}\right)$ & $\mathrm{PaO}_{2}(\mathrm{mmHg})$ & $\mathrm{PaCO}_{2}(\mathrm{mmHg})$ & $\mathrm{Arterial} \mathrm{pH}$ \\
\hline Baseline & $37.2 \pm 0.2$ & $96.6 \pm 5.4$ & $38.6 \pm 5.9$ & $7.37 \pm 0.08$ \\
Ischemia, 60 min & $37.7 \pm 0.3$ & $92.1 \pm 6.4$ & $37.3 \pm 3.8$ & $7.36 \pm 0.05$ \\
Reperfusion, 12 h & $37.5 \pm 0.2$ & $98.9 \pm 5.1$ & $38.1 \pm 4.1$ & $7.35 \pm 0.10$ \\
Reperfusion, 24 h & $37.1 \pm 0.1$ & $94.8 \pm 5.7$ & $35.6 \pm 4.3$ & $7.38 \pm 0.12$ \\
Reperfusion, 48 h & $37.3 \pm 0.2$ & $91.7 \pm 7.2$ & $37.8 \pm 5.2$ & $7.39 \pm 0.13$ \\
\hline
\end{tabular}

Results are presented as the mean $\pm \mathrm{SEM}(\mathrm{n}=6)$. $\mathrm{PaO}_{2}$, arterial oxygen tension; $\mathrm{PaCO}_{2}$, arterial carbon dioxide tension .

pole). The tissues were immediately frozen in liquid nitrogen and stored at $-80^{\circ} \mathrm{C}$. Total protein extraction was performed using a commercially available kit (KGP250; Nanjing Keygen Biotech Co. Ltd., Nanjing, China). Nuclear protein extraction was performed using the ProteoJET cytoplasmic and nuclear protein extraction kit (Fermentas International, Glen Burnie, MD, USA). Protein concentrations were determined using the BCA protein assay kit (Beyotime, Jiangsu, China).

Western blot analysis for aII-spectrin, TRPC6 and p-CREB. Protein samples from total or nuclear fractions were boiled for $10 \mathrm{~min}$ in $1 \mathrm{X}$ sample buffer (Beyotime) prior to loading onto a Tris- $\mathrm{HCl}$ gel. Equal amounts of total protein extracts or nuclear protein extracts were separated by SDS-PAGE and transferred onto polyvinylidene difluoride membranes by electrophoresis, and membranes were blocked with $5 \%$ non-fat milk in TBST (0.1\% Tween-20 in TBS) for $1 \mathrm{~h}$ at room temperature. Membranes were then incubated overnight at $4^{\circ} \mathrm{C}$ with either a mouse monoclonal anti-aII-spectrin (1:1000; Enzo Biochem, New York, USA), rabbit polyclonal anti-TRPC6 (1:1000; Abcam, Cambridge, MA, USA), rabbit polyclonal anti-p-CREB (1:1000; Cell Signaling Technology, Beverly, MA, USA), rabbit polyclonal anti-Lamin B1 (1:500; Bioworld Technology Inc., St. Louis, MN, USA) or mouse monoclonal anti-GAPDH antibody (1:100; Proteintech Group, Inc., Chicago, IL, USA) followed by horse radish peroxidase (HRP)-conjugated goat anti-mouse IgG antibody (1:3000; Proteintech Group, Inc.) or anti-rabbit IgG antibody (1:5000; Proteintech Group, Inc.). Labeled proteins were detected using the Chemi-Doc Imaging System (Bio-Rad, Hercules, CA, USA). Protein bands were quantified by Image $\mathrm{Lab}^{\mathrm{TM}}$ image acquisition and analysis software (Bio-Rad). Western blots were repeated three times using samples prepared from three different rats for each experimental condition studied.

Quantum dot-based immunofluorescence. At 12, 24 and $48 \mathrm{~h}$ after reperfusion, rats ( $\mathrm{n}=3$ for each group) were anesthetized with $10 \%$ chloral hydrate and infused through the left ventricle with cold saline as a vascular rinse followed by a fixing solution containing 4\% paraformaldehyde in PBS. The brains were removed and fixed overnight in $4 \%$ paraformaldehyde in PBS at $4^{\circ} \mathrm{C}$. The brains were then blocked and embedded in paraffin. Paraffin-embedded brains were cut into $4-\mu \mathrm{m}$ sections according to standard procedures. Paraffin sections ( $n=3$ for each group) were incubated overnight with antibodies against TRPC6 (1:100; Abcam) and p-CREB (1:100; Cell Signaling Technology) at $4^{\circ} \mathrm{C}$ after being blocked with
$2 \%$ bovine serum albumin (BSA). The samples were then incubated with a biotinylated secondary antibody at $37^{\circ} \mathrm{C}$ for $30 \mathrm{~min}$. Paraffin sections were then incubated with streptavidin-conjugated QDs605 (1:100, Wuhan Jiayuan Quantum Dot Co., Ltd., Wuhan, China) after being blocked with $2 \%$ BSA. TRPC6- and p-CREB-positive cells were measured at x200 magnification per visual field in the cortex, three visual fields per section in three brain sections. Fluorescent signals were detected with a fluorescence microscope (BX51; Olympus, Tokyo, Japan). The acquisition and quantitative analysis of images was performed with a multi-spectral imaging system (Nuance Fx; CRi, Hopkinton, MA, USA).

Statistical analysis. For all quantitative analysis of data, measurements were made with the experimenter blinded to the treatment group. GraphPad Prism (version 5 for Windows; GraphPad Software, La Jolla, CA, USA) software was used for all statistical analyses. Results are presented as the means \pm SEM. The neurological score data comparison was analyzed using the Kruskal-Wallis test followed by the post hoc Dunn's test. For all other measurements, one-way analysis of variance (ANOVA) followed by Newman-Keuls multiple comparison test was used. $\mathrm{P}<0.05$ was considered to indicate a statistically significant difference.

\section{Results}

Physiological parameters. No statistical significance was noted among different time points for any of the physiological parameters, including cranial temperature and blood gas (Table I).

NPD1 significantly reduces infarct volumes in ipsilateral ischemic hemispheres at $48 \mathrm{~h}$ after reperfusion. After ischemic/reperfusion injury, the white-stained infarct area was prominent in the MCAO group, and almost the entire MCA area appeared infarcted. By contrast, NPD1-treated rats had significantly reduced infarct volumes compared with the MCAO group $(\mathrm{P}<0.01)$. After application of PD98059, the infarct volume was significantly increased compared with the NPD1-treated group (Fig. 1A and B; $\mathrm{P}<0.01$ ).

NPD1 promotes functional recovery at $48 \mathrm{~h}$ after reperfusion. Sham surgery rats did not have any deficits. Statistical analysis confirmed that NPD1-treated animals had significantly greater neurological scores than the MCAO group $(\mathrm{P}<0.01)$. After 

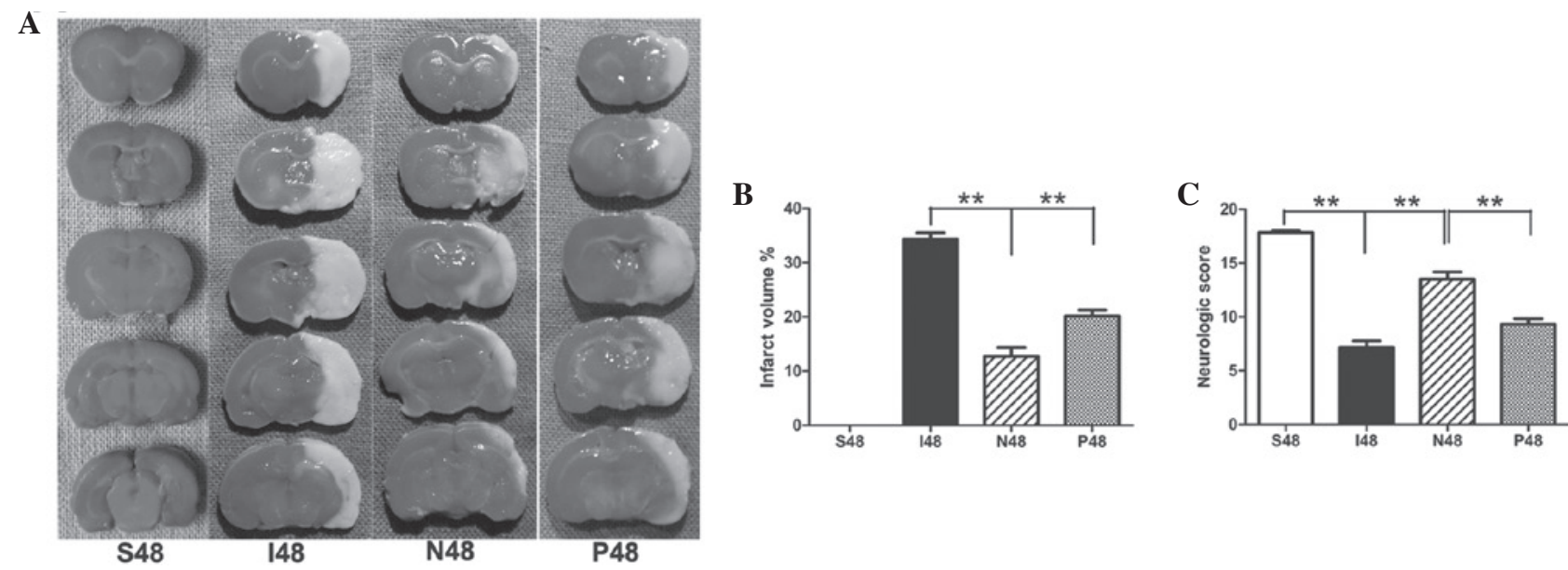

Figure 1. NPD1 reduced infarct volumes in the ipsilateral cortex and promoted functional recovery at $48 \mathrm{~h}$ after reperfusion. (A) TTC staining of representative coronal sections. (B) Quantification of infarct volumes. NPD1-treated rats had significantly reduced infarct volumes compared with the MCAO group $(\mathrm{P}<0.01)$. After application of PD98059, the infarct volume was significantly increased compared with the NPD1-treated group (P<0.01). Bars represent the means \pm SEM $(n=6)$. (C) Quantification of neurologic scores. NPD1-treated animals had significantly greater scores than the MCAO group. PD98059 administered 20 min prior to the surgery attenuated NPD1-induced functional recovery. Bars represent the means \pm SEM (n=6). ${ }^{* *} \mathrm{P}<0.01$. NPD1, neuroprotectin D1; MCAO, middle cerebral artery occlusion; TTC, 2,3,5-triphenyltetrazolium chloride

treatment with PD98059, the neurological scores were significantly decreased compared with the NPD1-treated group (Fig. 1C; $\mathrm{P}<0.01$ ).

NPD1 inhibits the formation of calpain-specific aII-spectrin breakdown products (SBDP145). The sham surgery group presented little SBDP145. Quantitative analysis confirmed that the protein levels of SBDP145 in the MCAO group were gradually increased during the experimental time course. Compared with the sham surgery group, the protein levels of SBDP145 in the MCAO group were significantly increased as early as $12 \mathrm{~h}$ after reperfusion indicating early calpain activity $(\mathrm{P}<0.05)$; this significant increase was also present at $24 \mathrm{~h}(\mathrm{P}<0.01)$ and $48 \mathrm{~h}(\mathrm{P}<0.01)$. When MCAO rats were treated with NPD1, the protein levels of SBDP145 were significantly decreased at $24 \mathrm{~h}$ $(\mathrm{P}<0.01)$ and $48 \mathrm{~h}(\mathrm{P}<0.01)$. In addition, NPD1 attenuated the calpain-specific fragment of aII-spectrin, but had no effect on caspase-3 and its cleavage activity on aII-spectrin (Fig. 2).

NPD1 inhibits calpain-mediated TRPC6 channel degradation. In the MCAO group, the TRPC6 protein level was also gradually decreased during the experimental time course (Fig. 3A and B). Compared with the sham surgery group, the TRPC6 level in the MCAO group was significantly decreased at $12 \mathrm{~h}(\mathrm{P}<0.01), 24 \mathrm{~h}(\mathrm{P}<0.01)$ and $48 \mathrm{~h}(\mathrm{P}<0.01)$. When MCAO rats were treated with NPD1, the protein levels of TRPC6 were significantly increased at $24 \mathrm{~h}(\mathrm{P}<0.05)$ and $48 \mathrm{~h}(\mathrm{P}<0.01)$. Immunofluorescence analysis showed the cytomembrane staining pattern of TRPC6 in neurons of the cerebral cortex and the immunofluorescence analysis obtained similar results as the western blot analysis (Fig. 3C and D).

PD98059 exerts no effect on ischemic stroke in rats at $24 \mathrm{~h}$ after reperfusion. To study the effect of PD98059 in strokeinduced rats, PD98059 was administered $20 \mathrm{~min}$ prior to surgery. Notably, after application of PD98059, no statistical significance was noted in the protein levels of $\mathrm{p}$-CREB between group I and group M (Fig. 4A). There was no significant difference between the two groups during the whole process of ischemia and reperfusion by measuring the infarct volumes and neurological scores (Fig. 4B and C).

NPD1 maintains phosphorylation of CREB through inhibition of TRPC6 degradation. In the MCAO group, p-CREB was gradually decreased during the experimental time course (Fig. 5A and B). Compared with the sham surgery group, p-CREB in the MCAO group was significantly decreased at $12 \mathrm{~h}(\mathrm{P}<0.01), 24 \mathrm{~h}(\mathrm{P}<0.01)$ and $48 \mathrm{~h}(\mathrm{P}<0.01)$. Compared with the MCAO group, $\mathrm{p}-\mathrm{CREB}$ in the NPD1-treated group was significantly higher than that at $24 \mathrm{~h}(\mathrm{P}<0.05)$ and $48 \mathrm{~h}$ $(\mathrm{P}<0.01)$. As expected, administration of PD98059 $20 \mathrm{~min}$ prior to surgery leads to a significantly decreased $\mathrm{p}-\mathrm{CREB}$ level compared with the NPD1-treated group at $12 \mathrm{~h}(\mathrm{P}<0.05)$, $24 \mathrm{~h}(\mathrm{P}<0.05)$ and $48 \mathrm{~h}(\mathrm{P}<0.01)$. Immunofluorescence staining also showed the same results, and immunoreactivity appeared as nucleus labeling, with no labeling within the cytoplasm or cell membrane (Fig. 5C and D).

\section{Discussion}

Our results strongly demonstrate that NPD1, when applied by the ICV route at $2 \mathrm{~h}$ after reperfusion, significantly reduced infarct volumes measured by TTC staining. We observed that the decreased infarct volumes obtained with NPD1 were mirrored by enhanced functional recovery. These protective effects are comparable with the observations of Marcheselli et al (12), who revealed that NPD1 administered continuously by ICV perfusion reduced the infarct volume by $50 \%$ at $48 \mathrm{~h}$ after reperfusion. In our study, application of NPD1 with PD98059 20 min prior to surgery led to infarct volumes that were significantly increased, and the neurological scores were significantly decreased. These results demonstrated that ICV injection of NPD1, at very low doses (500 ng, ICV), effectively reduced cerebral ischemic injury in rat models. 

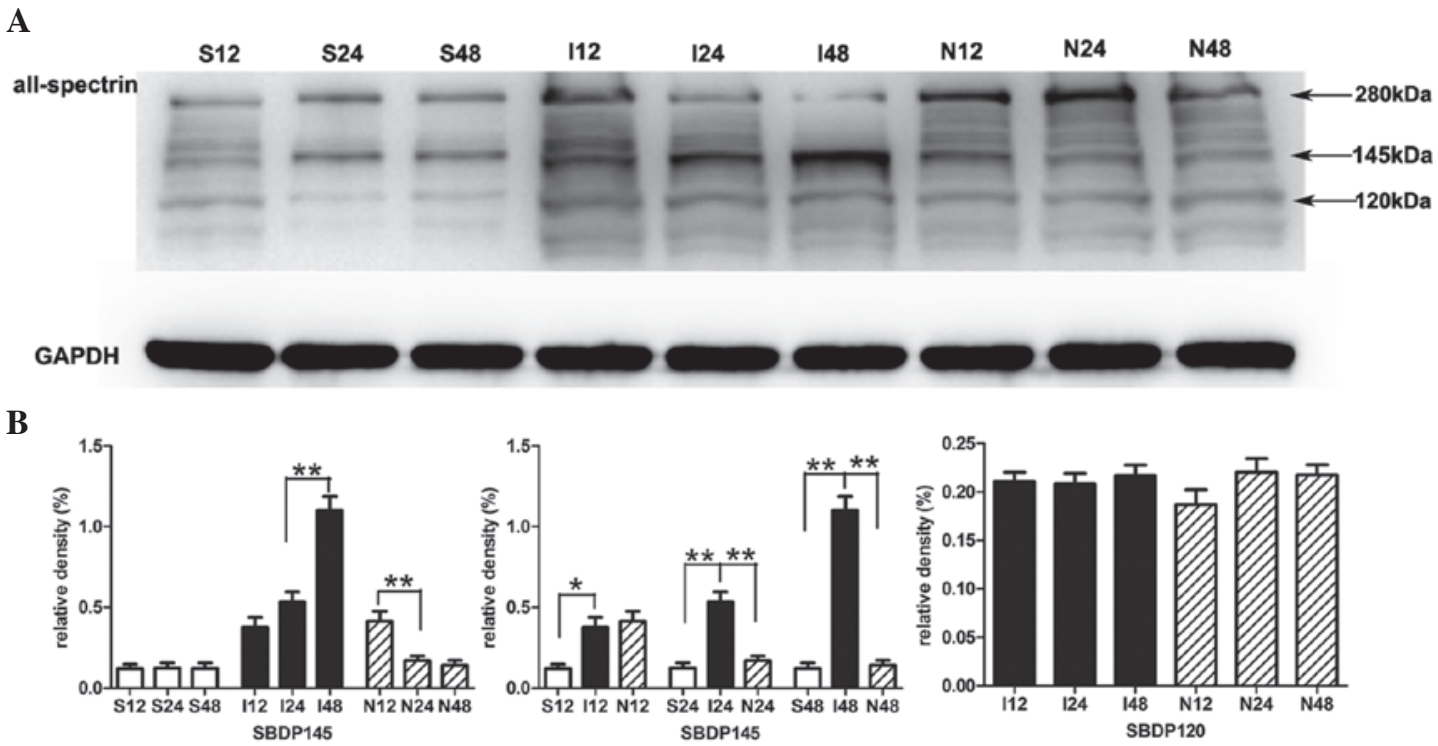

Figure 2. Calpain activation assessed by the protein levels of SBDP145. (A) Western blot analysis of SBDP145 expression at 12, 24 and $48 \mathrm{~h}$ after reperfusion. Total protein extracts were prepared from the ipsilateral cortex. Identical amounts of protein $(50 \mu \mathrm{g})$ were applied to each lane of the SDS-PAGE gel (6\% polyacrylamide gel). GAPDH was used as an internal reference. A 145-kDa band corresponding to SBDP145 protein was clearly detected. (B) Densitometric analysis of the protein levels of SBDP145 ( $\mathrm{n}=3)$. Application of NPD1 significantly reduced the protein levels of SBDP145 at $24 \mathrm{~h}(\mathrm{P}<0.01)$ and $48 \mathrm{~h}(\mathrm{P}<0.01)$ In addition, NPD1 attenuated calpain-specific fragment of aII-spectrin, but had no effect on caspase-3 and its cleavage activity on aII-spectrin. Values are the means \pm SEM. ${ }^{*} \mathrm{P}<0.05$ and ${ }^{* *} \mathrm{P}<0.01$. NPD1, neuroprotectin D1.

$\mathbf{A}$

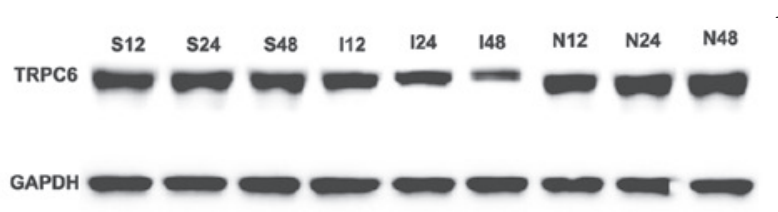

C

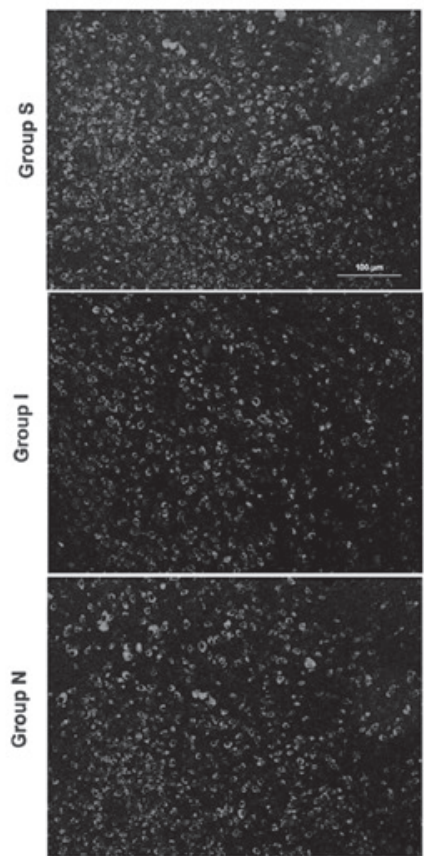

$12 \mathrm{~h} \mathrm{R}$
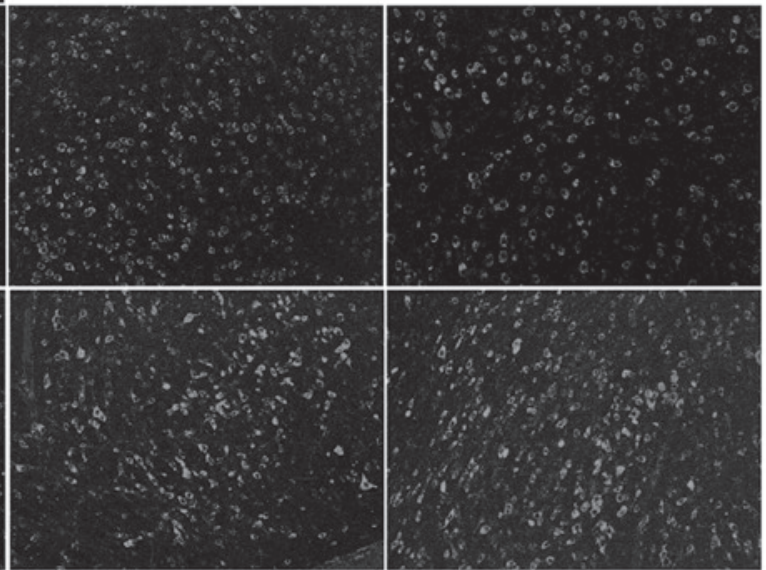

$24 \mathrm{~h} R$
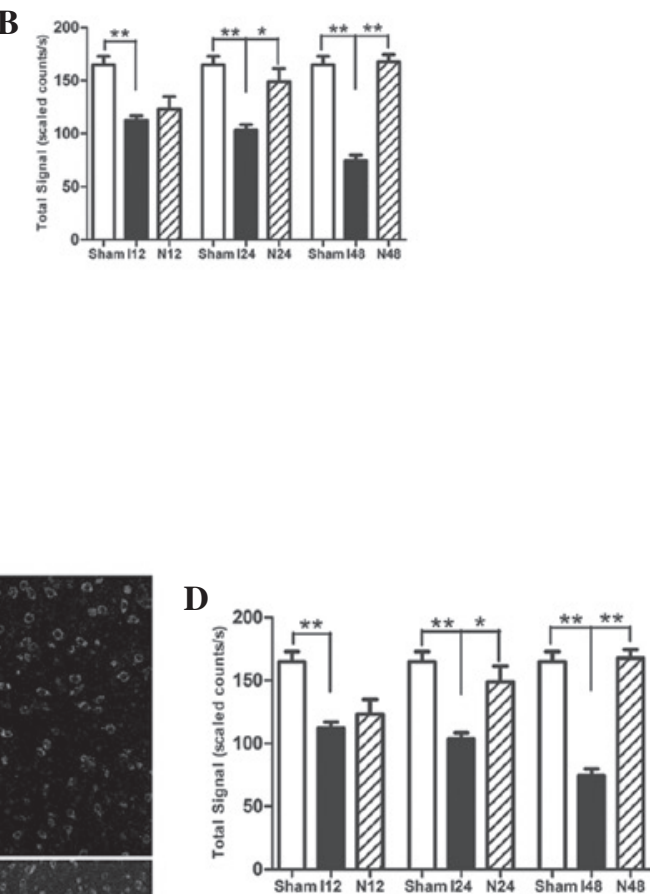

Figure 3. Effect of NPD1 administered at $2 \mathrm{~h}$ after reperfusion on TRPC6 expression at 12, 24 and $48 \mathrm{~h}$ after reperfusion. (A) Western blot analysis of TRPC6. Total protein extracts were prepared from the ipsilateral cortex. Identical amounts of homogenate protein $(50 \mu \mathrm{g})$ were applied to each lane of the SDS-PAGE gel (8\% polyacrylamide gel). GAPDH was used as an internal reference. A 106-kDa band corresponding to TRPC6 protein was clearly detected. (B) Densitometric analysis of the protein levels of TRPC6 $(\mathrm{n}=3)$. Application of NPD1 significantly reduced the protein levels of TRPC6 at $24 \mathrm{~h}(\mathrm{P}<0.05)$ and $48 \mathrm{~h}(\mathrm{P}<0.01)$. (C) TRPC6 immunoreactivity (x200). Staining was present within the cell membrane. (D) Quantification of the levels of TRPC6 ( $\mathrm{n}=3$ ). Values are the means \pm SEM. ${ }^{*} \mathrm{P}<0.05 ;{ }^{* *} \mathrm{P}<0.01$. NPD1, neuroprotectin D1; TRPC6, transient receptor potential canonical (subtype) 6. 
$\mathbf{A}$

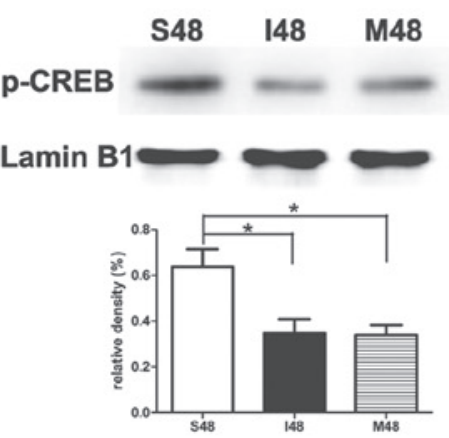

B

$\begin{array}{lll}548 & 148 \quad M 48\end{array}$
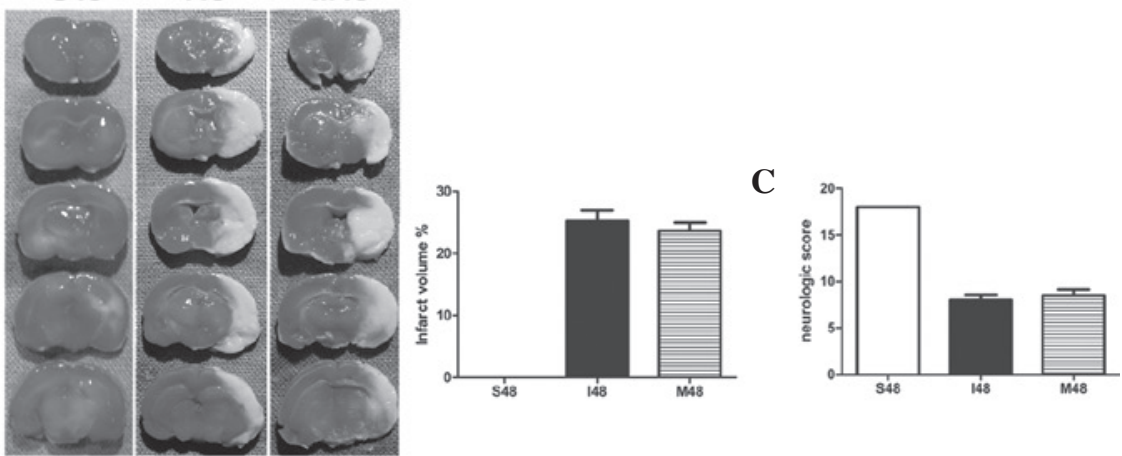

Figure 4. Effect of PD98059 on the stroke rats at $48 \mathrm{~h}$ after reperfusion. (A) Western blot analysis of p-CREB expression (n=3). Nuclear protein extracts were prepared from the ipsilateral cortex. Quantification of p-CREB assessed by western blot analysis was normalized to the expression level of Lamin B1. Application of PD98059, no statistical significance was noted in the protein levels of p-CREB in MCAO rats. (B) Representative TTC staining of the cerebral infarct in the rat brain $(n=6)$. (C) Quantification of neurological scores $(n=6)$. There was also no significant difference between group I and group M by measuring the infarct volumes and neurological scores. Values are the means \pm SEM. ${ }^{*} \mathrm{P}<0.05$ vs. sham surgery. p-CREB, phosphorylated cAMP-response element binding protein; MCAO, middle cerebral artery occlusion; TTC, 2,3,5-triphenyltetrazolium chloride.

A
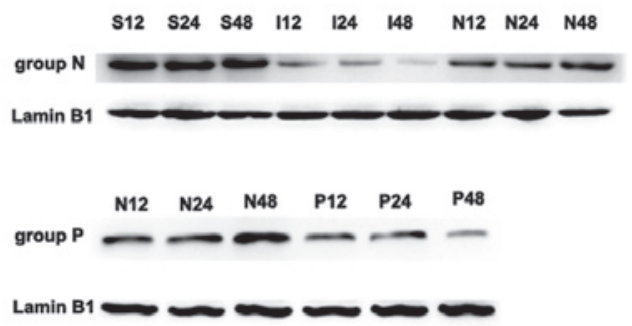

B
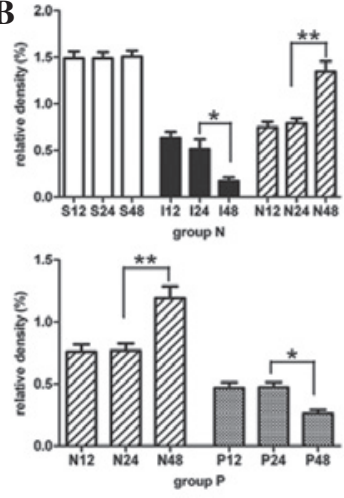
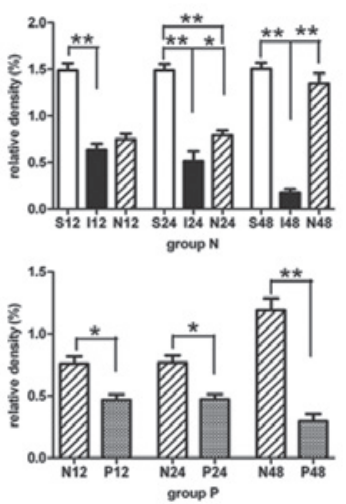

C

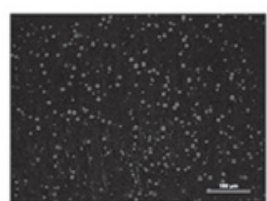

Group S
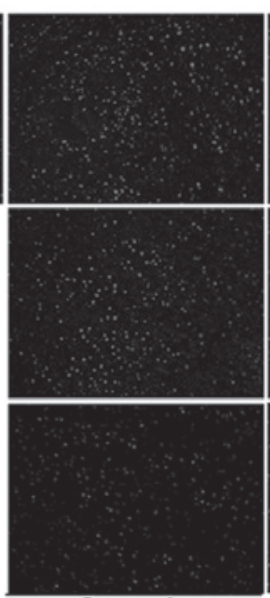

Group I
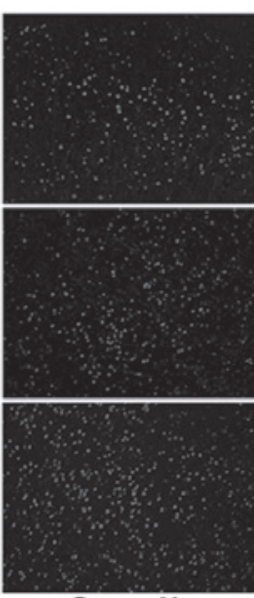

Group N

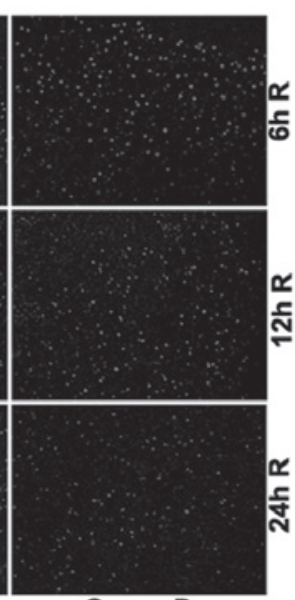

Group P

Figure 5. Effect of NPD1 administered at $2 \mathrm{~h}$ after reperfusion on the levels of p-CREB at 12, 24 and $48 \mathrm{~h}$ after reperfusion. (A) Western blot analysis of p-CREB expression. Nuclear protein extracts were prepared from the ipsilateral cortex. Identical amounts of homogenate protein $(60 \mu \mathrm{g})$ were applied to each lane of the SDS-PAGE gel (10\% polyacrylamide gel). Lamin B1 was used as an internal reference. A 43-kDa band corresponding to p-CREB protein was clearly detected. (B) Densitometric analysis of the protein levels of p-CREB ( $n=3)$. Application of NPD1 significantly increased the protein levels of $\mathrm{p}$-CREB in MCAO rats at $24 \mathrm{~h}(\mathrm{P}<0.05)$ and $48 \mathrm{~h}(\mathrm{P}<0.01)$. Following administration with PD98059 20 min prior to the surgery, the p-CREB level was significantly decreased at $12 \mathrm{~h}(\mathrm{P}<0.05), 24 \mathrm{~h}(\mathrm{P}<0.05)$ and $48 \mathrm{~h}(\mathrm{P}<0.01)$ compared with the NPD1-treated group. (C) $\mathrm{P}-\mathrm{CREB}$ immunoreactivity (x200). Staining was present within cell nucleus. (D) Quantification of the levels of $p-C R E B(n=3)$. Values are the means \pm SEM. $P<0.05$; ${ }^{* *} \mathrm{P}<0.01$. NPD1, neuroprotectin D1; p-CREB, phosphorylated $\mathrm{Ca}^{2+}$-response element binding protein; $\mathrm{MCAO}$, middle cerebral artery occlusion.

There are several mechanisms, including excitotoxicity, ionic imbalance, peri-infarct depolarizations, oxidative stresses and apoptosis $(1,2,5)$ that have been implicated in ischemic neuronal death. $\mathrm{Ca}^{2+}$ overload remains the most critical mechanism. The NMDA receptor is an important excitatory neurotransmitter receptor in the brain, which has been 
reported as the pivotal player for $\mathrm{Ca}^{2+}$ overload in response to cerebral ischemia. A large number of in vitro and in vivo studies have suggested that NMDA receptor antagonists are effective in ischemic neuronal death. Pharmacological agents that block glutamate release or glutamate-mediated postsynaptic excitability may reduce neural degeneration in stroke rats $(18,19)$. However, clinical trials examining the treatment of stroke using NMDA antagonists have all failed and have caused severe side effects (20).

Calpains are intracellular calcium-dependent cysteine endopeptidases, which are activated by NMDARs-mediated cytosolic $\mathrm{Ca}^{2+}$ overload $(8,21)$. Under physiological conditions, calpain activity is likely to be stimulated by transient localized increases in cytosolic $\mathrm{Ca}^{2+}$ and tightly regulated by the presence of an endogenous inhibitor calpastatin. By contrast, the increase in cytosolic $\mathrm{Ca}^{2+}$ during cerebral ischemia overwhelms endogenous regulatory systems resulting in pathological calpain activity $(6,22)$. Calpain inhibitors provide varying degrees of neuroprotection in animal models $(22,23)$. aII-Spectrin, the most well-studied target of calpain and caspase, is an abundant cytoskeletal protein that is specifically cleaved by calpain into $150 / 145-\mathrm{kDa}$, and is also specifically cleaved by caspase- 3 into $150 / 120-\mathrm{kDa}$ fragments. These characteristics make aII-spectrin cleavage a useful tool to evaluate the activity of calpains and caspase-3 (24,25). In our study, brain samples from sham surgery rats presented very little SBDP145, whereas MCAO rats had elevated levels of SBDP145 in the cortical regions of the ipsilateral hemisphere in the first $48 \mathrm{~h}$ post-injury. NPD1 treatment significantly reduced SBDP145 formation and made it recover to basal levels at $24 \mathrm{~h}$. However, NPD1 treatment had no effect on the formation of caspase-3-specific aII-spectrin breakdown products of $120 \mathrm{kDa}$ (SBDP120) in cerebral ischemia. Therefore, our results strongly demonstrate that NPD1, when applied at $2 \mathrm{~h}$ after reperfusion, specifically inhibited calpain (not caspase) activation, which induced resistance to ischemia and reperfusion injuries.

The transient receptor potential (TRP) channel was first identified in Drosophila melanogaster (26) and is a subfamily of the nonselective cation channels permeable to $\mathrm{Ca}^{2+}$. TRPC6 channels are present in numerous cell types, including neurons $(27,28)$. TRPC6 protein in neurons in ischemia was specifically downregulated by calpain proteolysis (8). Channels formed by the TRP family of proteins have a variety of biological functions. For example, TRPC3 and TRPC6 are involved in brain-derived neurotrophic factor (BDNF)-mediated growth cone turning, neuron survival and spine formation $(9,29)$. TRPC6 also promoted dendritic growth via the CaMKIV-CREB-dependent pathway (30). A previous study provided evidence that TRPC6 was specifically degraded in transient ischemia and this degradation occurred prior to and during neuronal cell death, and that increases in its protein level or activity prevented neuronal death. Therefore, the conventional conception about treatment of ischemic brain damage with NMDA receptor antagonists may have to be renovated. However, inhibition of calpain proteolysis of TRPC6 may protect animals from ischemic brain damage (8). TRPC6 channels play a critical role in promoting neuronal survival against focal cerebral ischemia and calpain-mediated downregulation of TRPC6 contributes to ischemic brain injury (8). In our study, the levels of TRPC6 proteins in the MCAO group were greatly decreased at $12 \mathrm{~h}$ after reperfusion and the reduction in TRPC6 protein levels remained prominent at 24 and $48 \mathrm{~h}$, in support of the observations of Du et al (8). NPD1 treatment significantly enhanced the protein levels of TRPC6 at 24 and $48 \mathrm{~h}$. In addition, NPD1 significantly reduced infarct volumes and enhanced functional recovery at $48 \mathrm{~h}$. Therefore, our results indicated that inhibition of calpain proteolysis of TRPC6 by NPD1 protects rats from ischemic brain damage.

In cortical neurons, entry of $\mathrm{Ca}^{2+}$ results in calciumdependent activation of ERK, which in turn activates CREB transcriptional pathways to support neuronal survival $(9,30,34)$. Phosphorylation of serine-133 in CREB allows it to contact its co-activator, CREB-binding protein/p300, and is necessary for its activation. The CREB activation is a critical event in neuroprotection against ischemic injury $(35,36)$. Overexpressing TRPC6 markedly increased CREB phosphorylation and CREB-dependent transcription (9). Blocking TRPC6 degradation maintained phosphorylation of CREB and greatly prevented ischemic brain damage. In our study, the protein levels of p-CREB significantly increased in the NPD1-treated group at $24 \mathrm{~h}$ and recovered to the level of the sham surgery group at $48 \mathrm{~h}$. When MEK activity was specifically inhibited by PD98059, the neuroprotective effect of NPD1 was attenuated and correlated with decreased CREB levels. These results clearly demonstrated that the activation of CREB through the MEK pathway is a pivotal downstream effector for the neuronal protective effect of TRPC6. Taken together, these results suggested that NPD1 blocked calpain-mediated TRPC6 channel degradation and stimulates the Ras/MEK/ERK pathway that converges on CREB activation, and contributed to neuroprotection.

Unlike the intravenous and intraperitoneal routes, the ICV route of administration is a useful experimental method to study the effects of chemicals or cellular grafts in the ventricular compartment of the brain following focal ischemia $(37,38)$. In the present study, it is noteworthy that ICV injection of NPD1 at $2 \mathrm{~h}$ after reperfusion very rapidly attenuated ischemic cerebral injury within $12 \mathrm{~h}$ of reperfusion. This rapid effect may be a result of the ICV route. The doses of NPD1 (500 ng) administered into the lateral ventricle were very low, consistent with one previous study (12), suggesting that ICV injection of NPD1 is a cost-effective and highly efficient method in cerebral ischemia.

In conclusion, our results suggest that ICV injection of NPD1 at very low doses (500 ng) significantly reduces calpain-mediated TRPC6 channel degradation, and stimulates the Ras/MEK/ERK pathway that converges on CREB activation and rapidly attenuates ischemic cerebral injury during the acute period of ischemic stroke. Therefore, ICV administration of NPD1 following cerebral ischemia as a neuroprotective treatment may confer clear advantages and provides theoretical support for the use of NPD1 in ischemic stroke management during the acute or subacute period.

\section{References}

1. Dirnagl U, Iadecola $\mathrm{C}$ and Moskowitz MA: Pathobiology of ischaemic stroke: an integrated view. Trends Neurosci 22: 391-397, 1999.

2. Lo EH, Dalkara T and Moskowitz MA: Mechanisms, challenges and opportunities in stroke. Nat Rev Neurosci 4: 399-415, 2003. 
3. Lo EH: A new penumbra: transitioning from injury into repair after stroke. Nat Med 14: 497-500, 2008.

4. Choi DW: Ischemia-induced neuronal apoptosis. Curr Opin Neurobiol 6: 667-672, 1996.

5. Lipton P: Ischemic cell death in brain neurons. Physiol Rev 79 1431-1568, 1999.

6. Bartus RT, Dean RL, Cavanaugh K, Eveleth D, Carriero DL and Lynch G: Time-related neuronal changes following middle cerebral artery occlusion: implications for therapeutic intervention and the role of calpain. J Cereb Blood Flow Metab 15 969-979, 1995.

7. Lipton SA and Rosenberg PA: Excitatory amino acids as a fina common pathway for neurologic disorders. N Engl J Med 330: 613-622, 1994.

8. Du W, Huang J, Yao H, Zhou K, Duan B and Wang Y: Inhibition of TRPC6 degradation suppresses ischemic brain damage in rats. J Clin Invest 120: 3480-3492, 2010.

9. Jia Y, Zhou J, Tai Y and Wang Y: TRPC channels promote cerebellar granule neuron survival. Nat Neurosci 10: 559-567, 2007.

10. Simopoulos AP: Omega-3 fatty acids, the brain and retina. Preface. World Rev Nutr Diet 99: VII-XII, 2009.

11. Niemoller TD, Stark DT and Bazan NG: Omega-3 fatty acid docosahexaenoic acid is the precursor of neuroprotectin D1 in the nervous system. World Rev Nutr Diet 99: 46-54, 2009.

12. Marcheselli VL, Hong S, Lukiw WJ, et al: Novel docosanoids inhibit brain ischemia-reperfusion-mediated leukocyte infiltration and pro-inflammatory gene expression. J Biol Chem 278 43807-43817, 2003.

13. Bazan NG: Cell survival matters: docosahexaenoic acid signaling, neuroprotection and photoreceptors. Trends Neurosci 29: 263-271, 2006.

14. Belayev L,Marcheselli VL, Khoutorova L, et al: Docosahexaenoic acid complexed to albumin elicits high-grade ischemic neuroprotection. Stroke 36: 118-123, 2005.

15. Rodriguez DTE, Belayev L, Liu Y, et al: Systemic fatty acid responses to transient focal cerebral ischemia: influence of neuroprotectant therapy with human albumin. J Neurochem 83 : 515-524, 2002

16. Bazan NG: Homeostatic regulation of photoreceptor cell integrity: significance of the potent mediator neuroprotectin D1 biosynthesized from docosahexaenoic acid: the Proctor Lecture. Invest Ophthalmol Vis Sci 48: 4866-4881, 4864-4865, 2007.

17. Tsubokawa T, Jadhav V, Solaroglu I, Shiokawa Y, Konishi Y and Zhang JH: Lecithinized superoxide dismutase improves outcomes and attenuates focal cerebral ischemic injury via antiapoptotic mechanisms in rats. Stroke 38: 1057-1062, 2007.

18. Shen H, Chen GJ, Harvey BK, Bickford PC and Wang Y: Inosine reduces ischemic brain injury in rats. Stroke 36: 654-659, 2005.
19. Shen H, Kuo CC, Chou J, et al: Astaxanthin reduces ischemic brain injury in adult rats. Faseb J 23: 1958-1968, 2009.

20. Hardingham GE, Fukunaga $\mathrm{Y}$ and Bading $\mathrm{H}$ : Extrasynaptic NMDARs oppose synaptic NMDARs by triggering CREB shut-off and cell death pathways. Nat Neurosci 5: 405-414, 2002.

21. Goll DE, Thompson VF, Li H, Wei W and Cong J: The calpain system. Physiol Rev 83: 731-801, 2003.

22. Yao H, Ginsberg MD, Eveleth DD, et al: Local cerebral glucose utilization and cytoskeletal proteolysis as indices of evolving focal ischemic injury in core and penumbra. J Cereb Blood Flow Metab 15: 398-408, 1995.

23. Hong SC, Goto Y, Lanzino G, Soleau S, Kassell NF and Lee KS: Neuroprotection with a calpain inhibitor in a model of focal cerebral ischemia. Stroke 25: 663-669, 1994.

24. Roberts-Lewis JM, Savage MJ, Marcy VR, Pinsker LR and Siman R: Immunolocalization of calpain I-mediated spectrin degradation to vulnerable neurons in the ischemic gerbil brain. J Neurosci 14: 3934-3944, 1994.

25. von Reyn CR, Spaethling JM, Mesfin MN, et al: Calpain mediates proteolysis of the voltage-gated sodium channel alpha-subunit. J Neurosci 29: 10350-10356, 2009.

26. Montell C, Jones K, Hafen E and Rubin G: Rescue of the Drosophila phototransduction mutation trp by germline transformation. Science 230: 1040-1043, 1985.

27. Harteneck C, Plant TD and Schultz G: From worm to man: three subfamilies of TRP channels. Trends Neurosci 23: 159-166, 2000.

28. Montell C, Birnbaumer L and Flockerzi V: The TRP channels, a remarkably functional family. Cell 108: 595-598, 2002.

29. Li Y, Jia YC, Cui K, et al: Essential role of TRPC channels in the guidance of nerve growth cones by brain-derived neurotrophic factor. Nature 434: 894-898, 2005.

30. Tai Y, Feng S, Ge R, et al: TRPC6 channels promote dendritic growth via the CaMKIV-CREB pathway. J Cell Sci 121: 2301-2307, 2008

31. Sossin WS and Barker PA: Something old, something new: BDNF-induced neuron survival requires TRPC channel function. Nat Neurosci 10: 537-538, 2007.

32. Walton MR and Dragunow I: Is CREB a key to neuronal survival? Trends Neurosci 23: 48-53, 2000.

33. Finkbeiner S: CREB couples neurotrophin signals to survival messages. Neuron 25: 11-14, 2000.

34. Chen J, Li Y and Chopp M: Intracerebral transplantation of bone marrow with BDNF after MCAo in rat. Neuropharmacology 39 711-716, 2000.

35. Modo M, Stroemer RP, Tang E, Patel S and Hodges H: Effects of implantation site of dead stem cells in rats with stroke damage. Neuroreport 14: 39-42, 2003. 